\title{
Impacts of restoration of an uncontrolled phosphogypsum dumpsite on the seasonal distribution of abiotic variables, phytoplankton, copepods, and ciliates in a man-made solar saltern
}

\author{
Rayda Kobbi-Rebai • Neila Annabi-Trabelsi • \\ Hajer Khemakhem • Habib Ayadi • Lotfi Aleya
}

Received: 6 January 2012 / Accepted: 10 May 2012

(C) Springer Science+Business Media B.V. 2012

\begin{abstract}
The restoration of an uncontrolled phosphogypsum landfill was investigated for its effects on the seasonal distribution of phytoplankton, ciliates, and copepods. Sampling was carried out monthly from September 2007 to August 2008 at four ponds of increasing salinity (A1, 41 psu; A5, 46 psu; A16, $67 \mathrm{psu}$; and C31, $77 \mathrm{psu}$ ) in the Sfax solar saltern (southeastern Tunisia). Physicochemical and biological analyses were carried out using standard methods. Results showed drastic reduction of phosphate input and greater diversity of phytoplankton, ciliates, and copepods than before restoration. Pennate diatoms and new ciliates, considered bio-indicators of less-stressed marine ecosystems, proliferated in the A1 pond for the first time after restoration. Copepods appeared to feed on a wide range of prey. Economically, removal of the 1.7 million $\mathrm{m}^{3}$ of phosphate improved the quality of
\end{abstract}

R. Kobbi-Rebai • N. Annabi-Trabelsi • H. Khemakhem •

H. Ayadi

Département des Sciences de la Vie,

Faculté des Sciences de Sfax, Université de Sfax, Unité de recherche UR/05ES05 Biodiversité et Ecosystèmes

Aquatiques, Route Soukra Km 3,5, B.P. 1171, 3000 Sfax,

Tunisia

L. Aleya $(\bowtie)$

Laboratoire de Chrono-Environnement, UMR CNRS 6249,

Université de Franche-Comté,

1, Place Leclerc,

25030 Besançon cedex, France

e-mail: lotfi.aleya@univ-fcomte.fr the site's salt production, enabling the salt company to receive the quality ISO 9001 accreditation.

Keywords Solar saltern · Phytoplankton ·

Ciliates · Copepods · Physicochemical variables .

Phosphogypsum restoration

\section{Introduction}

Coastal solar salterns are artificial and transitional ecosystems located between the marine environment and inland waters. They have been constructed in many areas worldwide and generally consist of a series of interconnected ponds featuring a salinity gradient ranging from near that of seawater level to a several-fold concentration ensuring a yield of halite used mainly as cooking salt and magnesium-rich bittern brines used to make road salt (Javor 1989, Khemakhem et al. 2010). Salterns are also biologically very rich, harboring birds, fish, plants and micro-organisms (Elevi-Bardavid et al. 2007; Abid et al. 2008). Despite their considerable landscape and economic value, and though studies clearly indicate that the salt-production process via evaporation and precipitation is closely related to biological processes (Davis 1990, 2000), salterns are now threatened biotopes, mainly due to human interference. This is particularly true of the Sfax saltern located on the southwestern Mediterranean coast and which suffered from pollution by phosphogypsum (Rekik et al. 2012) 
and petroleum products (Elloumi et al. 2008). Indeed, the anthropogenic nutrient input in the saltern's receiving pond may have impacted the development of the ponds biological communities and correlatively the quality of the salt produced (1990). It is therefore of interest to examine how algae, protozoans, and crustacean zooplankton, subjected to a long-term period of phosphogypsum-inducing stress, respond to improving ambient environmental conditions: persistence of the salt constraints in the saltern with the potential subsequent enhancement of the quality of the salt yield by approximately 300,000 tons of halite and 25,000 tons of bittern brine. In addition, the saltern had experienced the development of two green macroalgae (belonging especially to the genera Enteromorpha and Cladophora) and one red alga (Chondria) whose decomposition, along with that of the leaf waxes of land plants, had led to foul odors and an unpleasant appearance. The ponds, interconnected by pipes and channels along a 12-km stretch of seacoast, thus required significant expense and effort for maintenance and cleanliness (Elloumi et al. 2009). Consequently, Tunisian authorities undertook the Taparura project in 2006 to attack the phosphogypsum pollution at its source, to clean the ponds of an excess of macrophytes and to generally improve the regional environment. We (Abid et al. 2008; Elloumi et al. 2009; Khemakhem et al. 2010) and others (Joint et al. 2002; Oren 2005) have shown that salterns harbor a variety of organisms ranging from marine like to extreme halophiles and hypothesized that this restoration might lead to a change in the composition of the saltern's biological communities located near the phosphogypsum landfill. Salterns are, in some regards, well-studied systems (Oren 2005; Elloumi et al. 2009), yet aspects associated with anthropogenic impacts, especially from phosphogypsum residues on biological communities, have never before been reported. This lack of information may be explained by (1) the major cost and time constraints placed on this kind of study by the sorting and identification of the species present and (2) the hardship of discriminating between stress due to the salt gradient and that caused by phosphogypsum residues.

The present study is a part of an extensive research project undertaken to determine how the removal of nearly 1.7 million $\mathrm{m}^{3}$ of phosphate plate surrounding the landfill toe, and the dredging of nearly 0.5 million contaminated underwater samples, have affected the environmental quality in coastal ecosystems. The solar saltern of Sfax is a good example of this type of situation. From September 2007 to August 2008, we examined biological communities in four high-salinity lagoons, in order to detect how species richness and abundance compared along the salinity gradient (41$77 \mathrm{psu}$ ) during our post-restoration sampling period as opposed to pre-restoration data. Since the potential for niche partitioning based on the salinity gradient is greater for phytoplankton and ciliates than for copepods (Khemakhem et al. 2010), we chose this range of salinity so as to allow occurrence of all zooplankton, phytoplankton and ciliates as we have previously shown that within the 77 to below 120 salinity range, the copepod population, for example, would be marked by the presence of only two species namely Bryocamptus sp. and Mesochra lilljeborgi (Khemakhem et al. 2010). Finally, and since very few saltern studies have simultaneously considered many compartments within the plankton community, we attempted to assess, after restoration, the relationships between copepods and their potential prey such as phytoplankton and ciliates, which are currently hypothesized to be advantaged in such systems (Khemakhem et al. 2010).

\section{Materials and methods}

\section{Study site}

The solar saltern under study is located in the southern part of the city of Sfax along the east-central coast of Tunisia (between $34^{\circ} 39^{\prime} 0.1^{\prime \prime} \mathrm{N}$ and $10^{\circ} 42^{\prime} 35^{\prime \prime} \mathrm{E}$ ) extending over an area of nearly 1,500 ha. These manmade systems are formed by ponds (20-70 cm depth and 37-400 psu) connected by pipes and channels along a $12-\mathrm{km}$ section of seacoast. Of increasing salinity, ranging from that of seawater to that of crystallizers, the ponds attain a salt concentration rarely seen in natural environments in which halite (used mainly as cooking salt) is produced and where magnesium-rich bittern brines are collected (Elloumi et al. 2008) (Fig. 1). The saltern is separated from the sea by an artificial red silt seawall (height, $4 \mathrm{~m}$ ). Depending on meteorological conditions, seawater influx and circulation between the ponds are entirely controlled in order to ensure an optimal yield of halite and bittern brine.

Sampling and laboratory analyses

Sampling was carried out monthly from September 2007 to August 2008 in the four ponds whose salinity 


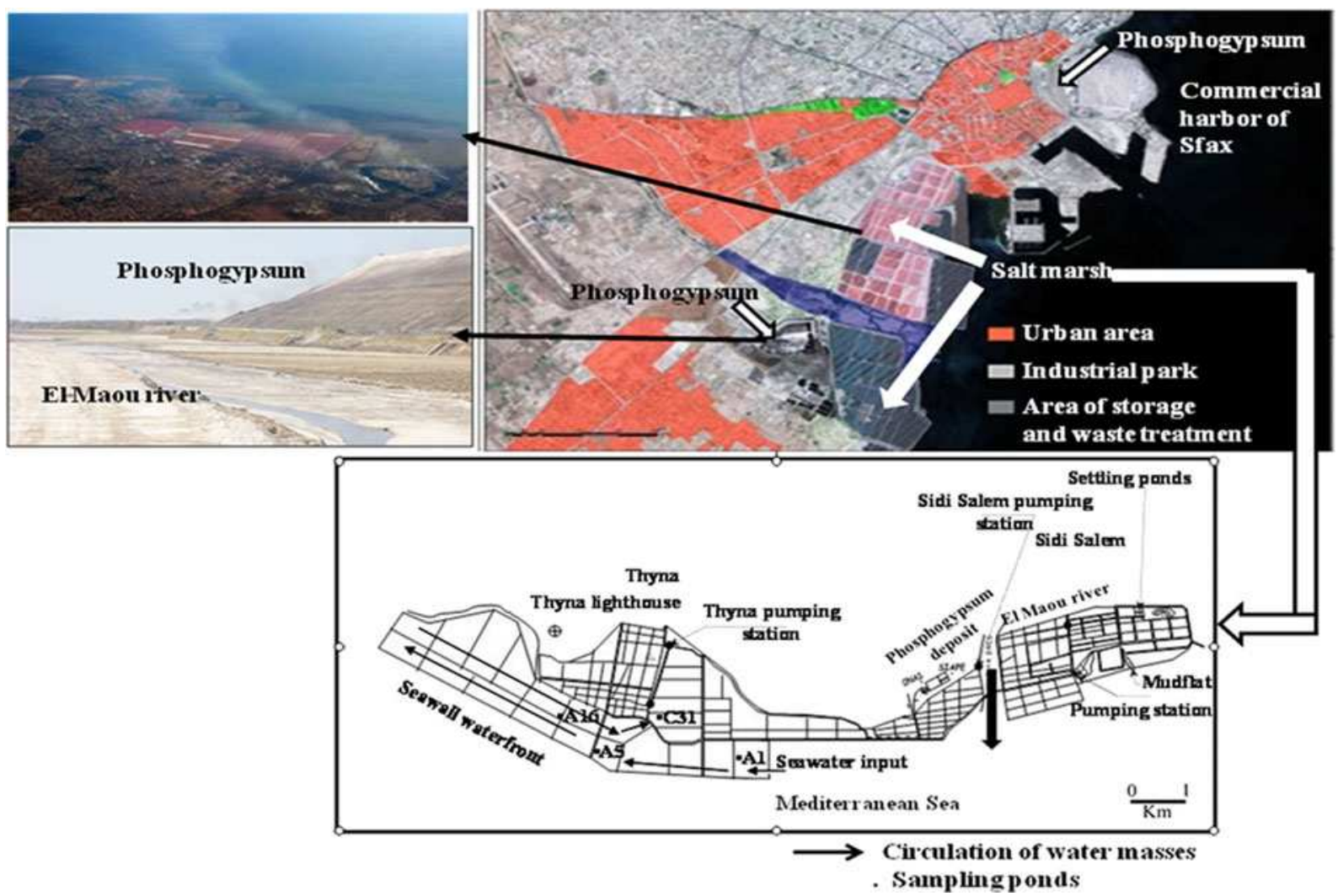

Fig. 1 Circulation of water masses and location of the sampling ponds A1, A5, A16, and C31 and phosphogypsum deposited near the Sfax solar saltern

increased as follows: A1, 41 psu; A5, 46 psu; A16, $67 \mathrm{psu}$; and $\mathrm{C} 31,77 \mathrm{psu}$. The seasons were defined as (1) autumn, September to November 2007; (2) winter, December 2007 and January-February 2008, (3) spring, March to May 2008, and (4) summer, June to August 2008. Water samples were collected at 10 $20 \mathrm{~cm}$ depth with a 1-1 Van Dorn bottle.

Temperature and $\mathrm{pH}$ were measured in situ using a mercury glass thermometer graduated in $0.1^{\circ} \mathrm{C}$ and a Metrohm $^{\circledR}$ type $\mathrm{pH}$ meter. Salinity, as totally dissolved salts, were estimated by the dry residue method, which consists of evaporating a $50 \mathrm{ml}$ sample $\left(24 \mathrm{~h}, 180^{\circ} \mathrm{C}\right)$ in a previously sterilized crystallizing dish (by heating at $550^{\circ} \mathrm{C}$ for $1 \mathrm{~h}$ ), and calculating the salt content from the difference in weight before and after evaporation (Elloumi et al. 2009). Concentration of suspended matter was determined by measuring the dry weight of the residue after water filtration through a Whatman $\mathrm{GF} / \mathrm{C}$ membrane. Total nitrogen and total phosphate (T-P) concentrations were assessed (after transformation into $\mathrm{NH}_{4}{ }^{+}$and $\mathrm{PO}_{4}{ }^{3-}$, with nitrogen persulfate and potassium persulfate, respectively at $120^{\circ} \mathrm{C}$ ) by spectrophotometry (APHA 2012). Nitrates were assayed by a Shimadzu ion chromatograph HIC-6A, equipped with an ion-exchange resin (i.e., the stationary phase) that is extremely stable at a wide range of salt concentrations and adapts well to a $\mathrm{pH}$ range of 212 (detection limit for $\mathrm{NO}_{3}{ }^{-}, 0.07 \mu \mathrm{mol} 1^{-1}$ ).

Water samples $(200 \mathrm{ml})$, taken in duplicate for phytoplankton and ciliates, were fixed with acid Lugol's iodine ( $1 \%$ final concentration) and stored in the dark at $4{ }^{\circ} \mathrm{C}$ until laboratory analysis. Phytoplankton and ciliates were counted under an inverted microscope $(\times 400)$ using the Uthermöl (1958) method. Chlorophyll $a$ was extracted using $10 \mathrm{ml}$ of acetone and determined spectrophotometrically after filtering a $0.15-1$ sample through a Whatman $\mathrm{GF} / \mathrm{C}$ filter. Concentrations were then calculated according to Strickland and Parsons (1968) equations. Crustacean zooplankton, collected by filtering 501 of water through a $50-\mu \mathrm{m}$ mesh net, were transferred to a $125-\mathrm{ml}$ flask and fixed with formaldehyde (5\%). For each sample, the community structure was determined by 
the Shannon-Weaver diversity index $\left(\mathrm{H}^{\prime}\right)$ (Shannon and Weaver 1949).

\section{Statistics}

Mean and standard deviation were reported when appropriate. One-way ANOVA analysis, followed by a post-hoc comparison using Tukey's test, was applied to identify significant differences in physicochemical and biological parameters among the ponds. ANOVA tests were undertaken using Excel-Stat software. Prior to the ANOVA tests, the normality of all variables was checked by means of the Kolmogorov-Smirnov test and no departure from normality was detected for any variable. Pearson's correlation analysis was performed to evaluate potential relationships between copepod abundance and abiotic and biotic variables. The data recorded in this study were examined with principal component analysis (PCA) in order to relate the distribution of copepods to the variables under study.

\section{Results}

Physicochemical parameters

Salinity, temperature, suspended matter and nutrient ranges are given in Table 1. Due to the shallowness of the four sampled ponds, no thermal stratification occurred. Water temperature differed slightly from pond to pond, with seasonal variations similar in all ponds (Table 1), varying from 11.5 (A1, January 2008) to $30^{\circ} \mathrm{C}$ (A16, September 2007) and correlating negatively with salinity, especially from April to September. This paradox is explained by the opening of water gates by the saltern's managers to feed the ponds with seawater. The annual average of N/P, expressed as DIN/DIP ratio where DIN is dissolved inorganic nitrogen $\left(\mathrm{DIN}=\mathrm{NO}_{2}{ }^{-}+\mathrm{NO}_{3}{ }^{-}+\mathrm{NH}_{4}{ }^{+}\right)$and DIP is dissolved inorganic phosphorus ( $\mathrm{DIP}=\mathrm{PO}_{4}{ }^{3-}$ ), which is the indicator of nutrient limitation for phytoplankton, ranged from 0.51 at $\mathrm{A} 1$ to 0.80 at $\mathrm{A} 16$. These averages were less than the Redfield ratio (16), suggesting a potential $\mathrm{N}$ limitation.

\section{Phytoplankton}

In this study, we identified a total of 72 phytoplankton taxa including diatoms, dinoflagellates, Cyanobacteriae, Euglenophyceae, and silicoflagellates. The mean values of phytoplankton abundance at each pond did not exceed

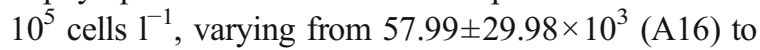
$85.44 \pm 26.58 \times 10^{3}$ cells $^{-1}$ (A5) (Fig. 2; Table 2). Diatoms dominated phytoplankton abundances in ponds A1 and A5 (salinity, $<50 \mathrm{psu}$ ) while dinoflagellates dominated in ponds with salinityof $>50$ psu (Fig. 2). Abundance of both diatoms and dinoflagellates were significantly different from pond to pond (ANOVA, $p<0.001$ ). During the sampling period, phytoplankton diversity was greater in pond A1 due to a highly diverse diatom community (Table 2).

Chlorophyll $a$ concentrations ranged from 0.055 (A1, June 2008) to $0.187 \mathrm{mg} \mathrm{l}^{-1}$ (C31, June 2008). Their temporal variation showed a maximum in March
Table 1 Annual mean \pm SD of physicochemical variables at ponds A1, A5, A16, and C31

In the last column, results of one-way ANOVA analysis. $F$ values-between groups mean square/within-groups mean square

$* p<0.05 ; * * p<0.01$;

$* * * p<0.001$, significant difference between sampled ponds

\begin{tabular}{|c|c|c|c|c|c|}
\hline \multirow{2}{*}{$\begin{array}{l}\text { Physicochemical } \\
\text { parameters (mean } \pm \mathrm{SD} \text { ) }\end{array}$} & \multicolumn{5}{|l|}{ Ponds } \\
\hline & A1 & A5 & A16 & $\mathrm{C} 31$ & $F$ value $(d f)$ \\
\hline \multicolumn{6}{|l|}{ Physical parameters } \\
\hline Salinity (psu) & $41.5 \pm 2.3$ & $46.4 \pm 5.2$ & $67.6 \pm 7.2$ & $77.0 \pm 4.3$ & $129.62(44)^{* * *}$ \\
\hline Temperature $\left({ }^{\circ} \mathrm{C}\right)$ & $20.7 \pm 5.5$ & $21.1 \pm 5.5$ & $20.9 \pm 5.8$ & $21.2 \pm 6.1$ & $0.02(44)$ \\
\hline $\mathrm{SM}\left(\times 10^{3} \mathrm{mg}^{-1}\right)$ & $1.1 \pm 0.5$ & $1.2 \pm 1.1$ & $1.1 \pm 0.9$ & $1.3 \pm 0.5$ & $0.20(44)$ \\
\hline \multicolumn{6}{|l|}{ Chemical parameters } \\
\hline $\mathrm{NO}_{2}^{-}\left(\mu \mathrm{mol} \mathrm{l}{ }^{-1}\right)$ & $1.3 \pm 2.2$ & $0.2 \pm 0.2$ & $0.3 \pm 0.1$ & $0.6 \pm 0.3$ & $2.14(44)$ \\
\hline $\mathrm{NO}_{3}^{-}\left(\mu \mathrm{mol} \mathrm{l}{ }^{-1}\right)$ & $2.3 \pm 1.2$ & $6.5 \pm 3.7$ & $7.1 \pm 5.4$ & $6.8 \pm 6.7$ & $2.63(44)$ \\
\hline $\mathrm{NH}_{4}^{+}\left(\mu \mathrm{mol} \mathrm{l}{ }^{-1}\right)$ & $6.3 \pm 5.7$ & $5.2 \pm 5.8$ & $5.3 \pm 4.5$ & $5.7 \pm 7.8$ & $0.08(44)$ \\
\hline $\mathrm{PO}_{4}{ }^{3-}\left(\mu \mathrm{mol} 1^{-1}\right)$ & $19.1 \pm 11.9$ & $15.3 \pm 5$ & $15.8 \pm 11.5$ & $17.5 \pm 13.8$ & $0.29(44)$ \\
\hline $\mathrm{T}-\mathrm{N}\left(\mu \mathrm{mol} \mathrm{l} 1^{-1}\right)$ & $16.3 \pm 7.7$ & $6.65 \pm 11.6$ & $13.2 \pm 15.9$ & $17.0 \pm 11.8$ & $1.82(44)$ \\
\hline T-P $\left(\mu \mathrm{mol} \mathrm{1} 1^{-1}\right)$ & $60.5 \pm 57.3$ & $9.0 \pm 4.78$ & $8.6 \pm 7.0$ & $14.5 \pm 11.2$ & $8.62(44) * * *$ \\
\hline
\end{tabular}


Fig. 2 Temporal and spatial variation of average phytoplankton abundances (a), ciliates (b), the total zooplankton, and total copepods (c) in ponds A1, A5, A16, and $\mathrm{C} 31$
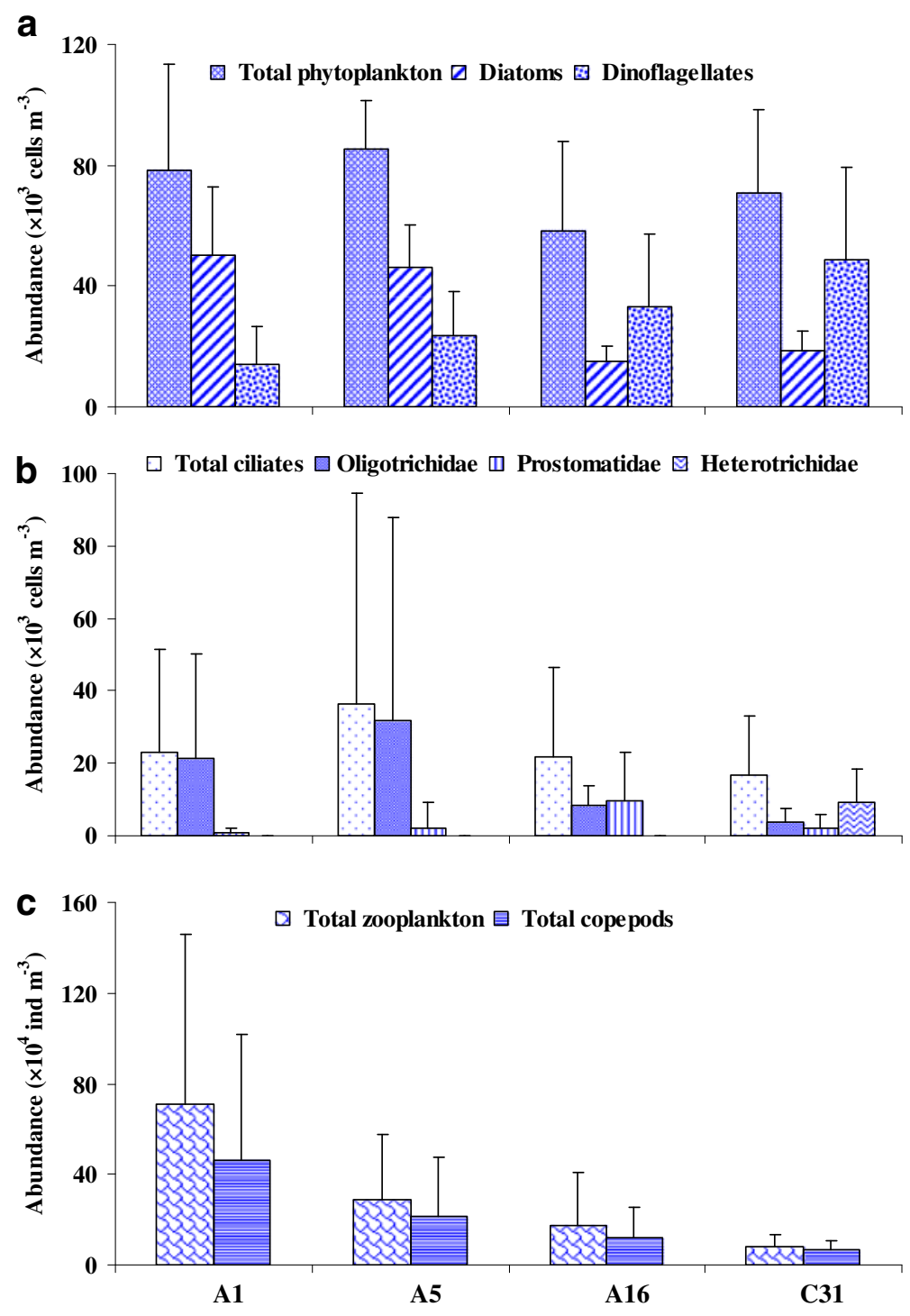

2008 in the first three ponds while the highest value was recorded in June 2008 at pond C31, (Fig. 3).

\section{Ciliates}

The ciliate community was composed of nine groups dominated by Oligotrichidae, Prostomatidae, and Heterotrichidae, the latter observed only in pond C31 (Fig. 2). Other groups, namely Hypotrichidae, Gymnostomatidae, Pleuronematina, Colpodea, Hymenostomatidae, and Peritrichidae were numerically inferior. Average total ciliate abundances varied from $16.65 \pm$ $16.31 \times 10^{3}$ (C31) to $36.26 \pm 58.25 \times 10^{3}$ (A5) cells $1^{-1}$ (Fig. 2; Table 3). Oligotrichidae dominated the ciliate abundance at ponds with less than 50 psu (A1 and A5). Annual mean values of $\mathrm{H}^{\prime}$ showed a general decrease according to salinity, the highest being 1.79 bits cell $^{-1}$ at A1 and the lowest, 1.36 bits cell $^{-1}$, at C31 (Table 3).

Copepod community composition and dynamics

A total of 17 taxa belonging to four orders (Calanoida, Cyclopoida, Harpacticoida, and Poecilostomatida) were distinguished. Ten taxa were found at all ponds. Harpacticoida dominated the copepod assemblage at C31 on all sampling dates. Cyclopoida dominated during winter, spring and summer at $\mathrm{A} 5$ and during autumn, winter and summer at A16. Calanoida 
Table 2 Quantitative and structural characteristics of the phytoplankton community sampled in ponds A1, A5, A16, and C31

\begin{tabular}{|c|c|c|c|c|c|}
\hline $\begin{array}{l}\text { Total phytoplankton } \\
\text { abundance } \\
\left(\times 10^{3} \text { cell } 1^{-1}\right)\end{array}$ & $\begin{array}{l}\text { Diversity index } \\
\left(\text { bits cell }{ }^{-1} \text { ) }\right.\end{array}$ & Evenness & $\begin{array}{l}\text { Abundance of } \\
\text { dominant species } \\
\left(\times 10^{3} \text { cell } 1^{-1}\right)\end{array}$ & $\begin{array}{l}\text { Occurrence frequency of } \\
\text { dominant species }(\%)\end{array}$ & Dominant species \\
\hline
\end{tabular}

A1

\begin{tabular}{|c|c|c|c|c|c|c|}
\hline $13 / 09 / 2007$ & 143.2 & 2.71 & 0.15 & 49.53 & 41.66 & Thalassiosira $\mathrm{sp}$. \\
\hline 29/10/2007 & 99.5 & 3.64 & 0.21 & 21 & 8.33 & Oscillatoria sp. \\
\hline $27 / 11 / 2007$ & 104 & 3.21 & 0.19 & 33 & 33.33 & Eutreptia globulifera \\
\hline $12 / 12 / 2007$ & 66.5 & 3.67 & 0.22 & 16.5 & & E. globulifera \\
\hline 28/01/2011 & 29 & 2.1 & 0.14 & 14 & 83.33 & Nitzschia longissima \\
\hline 02/02/2011 & 33 & 3.73 & 0.24 & 12.2 & & N. longissima \\
\hline 04/03/2011 & 37 & 2.67 & 0.17 & 11.2 & 41.66 & Prorocentrum triestinum \\
\hline 04/04/2011 & 89 & 2.65 & 0.16 & 35 & & E. globulifera \\
\hline 05/05/2011 & 92.5 & 3.51 & 0.21 & 17.5 & & E. globulifera \\
\hline 06/06/2011 & 96 & 2.66 & 0.16 & 26 & 25 & Euglena sp. \\
\hline 23/07/2011 & 54.8 & 1.59 & 0.1 & 37.6 & & N. longissima \\
\hline 08/08/2011 & 99 & 2.88 & 0.17 & 32.77 & & N. longissima \\
\hline \multicolumn{7}{|l|}{ A5 } \\
\hline $13 / 09 / 2007$ & 82.3 & 1.62 & 0.32 & 48.6 & 100 & N. longissima \\
\hline $29 / 10 / 2007$ & 88.4 & 2.29 & 0.46 & 42 & 66.66 & Prorocentrum micans \\
\hline $27 / 11 / 2007$ & 96 & 3.1 & 0.62 & 21 & & P. micans \\
\hline $12 / 12 / 2007$ & 97.55 & 0.91 & 0.18 & 26.25 & 58.33 & E. globulifera \\
\hline $28 / 01 / 2011$ & 99.1 & 0.72 & 0.14 & 35 & & E. globulifera \\
\hline $02 / 02 / 2011$ & 71.95 & 0.85 & 0.17 & 19 & 91.66 & Navicula sp. \\
\hline 04/03/2011 & 44.8 & 0.66 & 0.14 & 18 & & Navicula sp. \\
\hline 04/04/2011 & 97 & 0.72 & 0.14 & 47 & 16.66 & Euglena sp. \\
\hline 05/05/2011 & 103.5 & 0.99 & 0.19 & 29 & & N. longissima \\
\hline 06/06/2011 & 110 & 0.79 & 0.15 & 53 & & N. longissima \\
\hline $23 / 07 / 2011$ & 76 & 0.49 & 0.10 & 55.2 & & N. longissima \\
\hline 08/08/2011 & 79.15 & 0.58 & 0.11 & 51.9 & & N. longissima \\
\hline \multicolumn{7}{|l|}{ A16 } \\
\hline $13 / 09 / 2007$ & 27.2 & 0.69 & 0.14 & 13 & 100 & Nitzschia sp. \\
\hline $29 / 10 / 2007$ & 38.14 & 1 & 0.2 & 10 & & Nitzschia sp. \\
\hline $27 / 11 / 2007$ & 43.95 & 0.82 & 0.16 & 19 & 50 & Oxyrrhis marina \\
\hline $12 / 12 / 2007$ & 57.37 & 0.78 & 0.15 & 25 & & O. marina \\
\hline 28/01/2011 & 70.8 & 0.69 & 0.13 & 31 & & O. marina \\
\hline $02 / 02 / 2011$ & 75.42 & 0.72 & 0.13 & 40.25 & & O. marina \\
\hline 04/03/2011 & 80.05 & 0.61 & 0.11 & 49.5 & & O. marina \\
\hline $04 / 04 / 2011$ & 77.2 & 0.71 & 0.13 & 31 & 66.66 & Euglena sp. \\
\hline $05 / 05 / 2011$ & 88.15 & 0.95 & 0.18 & 18.5 & & Nitzschia sp. \\
\hline 06/06/2011 & 99.1 & 0.77 & 0.14 & 36 & 16.66 & Oscillatoria sp. \\
\hline $23 / 07 / 2011$ & 9.5 & 0.81 & 0.18 & 4 & & Nitzschia $\mathrm{sp}$. \\
\hline 08/08/2011 & 18.35 & 0.84 & 0.18 & 8.5 & & Nitzschia sp. \\
\hline \multicolumn{7}{|l|}{$\mathrm{C} 31$} \\
\hline $13 / 09 / 2007$ & 129.5 & 0.92 & 0.17 & 36.5 & 91.66 & P. micans \\
\hline 29/10/2007 & 71.47 & 0.97 & 0.19 & 15.6 & 66.66 & Oxyrhiss marina \\
\hline $27 / 11 / 2007$ & 76 & 1.03 & 0.21 & 21 & & O. marina \\
\hline
\end{tabular}


Table 2 (continued)

\begin{tabular}{lllllll}
\hline & $\begin{array}{l}\text { Total phytoplankton } \\
\text { abundance } \\
\left(\times 10^{3} \text { cell } 1^{-1}\right)\end{array}$ & $\begin{array}{l}\text { Diversity index } \\
\left(\text { bits cell }{ }^{-1}\right)\end{array}$ & Evenness & $\begin{array}{l}\text { Abundance of } \\
\text { dominant species } \\
\left(\times 10^{3} \text { cell } 1^{-1}\right)\end{array}$ & $\begin{array}{l}\text { Occurrence frequency of } \\
\text { dominant species }(\%)\end{array}$ & Dominant species \\
\hline $12 / 12 / 2007$ & 55 & 1.19 & 0.25 & 12.75 & & O. marina \\
$28 / 01 / 2011$ & 34 & 1.05 & 0.23 & 6.5 & 25 & Gymnodinium nagasakiens \\
$02 / 02 / 2011$ & 72.69 & 0.99 & 0.20 & 25.4 & & O. marina \\
$04 / 03 / 2011$ & 111.39 & 0.79 & 0.15 & 46.3 & 16.66 & O. marina \\
$04 / 04 / 2011$ & 60.1 & 0.92 & 0.19 & 20 & & Prorocentrum lima \\
$05 / 05 / 2011$ & 54.97 & 1.06 & 0.22 & 10 & 66.66 & Gymnodinium sp. \\
$06 / 06 / 2011$ & 49.84 & 0.86 & 0.18 & 12 & 16.66 & Amphiporora sp. \\
$23 / 07 / 2011$ & 44.7 & 1.06 & 0.22 & 9 & & P. micans \\
$08 / 08 / 2011$ & 87.1 & 1.07 & 0.21 & 21.25 & & \\
\hline
\end{tabular}

dominated the copepod assemblage at A1, in March 2008 and at A5 in September 2007 and March 2008 (Figs. 2 and 4). Oithona nana (Cyclopoida) was the most abundant copepod at ponds A1, A5 and A16 (Table 4). Bryocamptus sp. (Harpacticoida) was the most abundant copepod at $\mathrm{C} 31$. The seasonal distribution of the copepod is presented in Fig. 4 and Table 4. Seasonal averages of $\mathrm{H}^{\prime}$ showed a general decrease as salinity increased from $\mathrm{A} 1$ to $\mathrm{C} 31 . \mathrm{H}^{\prime}$ varied from 1.4 (winter, C31) to 3.2 bits ind $^{-1}$ (winter, A1) (Fig. 5).

Average annual abundance of total zooplankton decreased from $75.50 \times 10^{4}$ ind $\mathrm{m}^{-3}$ at $\mathrm{A} 1$ to $5.46 \times$ $10^{4}$ ind $\mathrm{m}^{-3}$ at $\mathrm{C} 31$ parallel with the increase in pond salinity. Copepods were the dominant zooplankton (Fig. 2) accounting for 66.59, 67.51, 73.27, and $82.05 \%$ of total zooplankton abundance in A1, A5, A16, and C31, respectively. The monthly variation of copepod abundance clearly differed among the ponds. However, two peaks were observed during the same period (October 2007 and July 2008) for ponds A5 and C31 (Fig. 4). Only one major peak of copepod abundance was observed in A1 $\left(1.8 \times 10^{6}\right.$ ind $\mathrm{m}^{-3}$, January 2008) (Fig. 4).

\section{Principal component analysis}

Component axes $F 1$ and $F 2$ explained $84.29 \%$ of the variance (Fig. 6), with the former explaining $55.87 \%$. The $F 1$ axis shows negative correlation of salinity to total phytoplankton, diatoms, oligotrochida, total zooplankton, total copepods and thalassophylic copepods. Total copepods and total zooplankton also showed no negative correlation with $\mathrm{NO}_{3}{ }^{-}$. The three species from marine contamination and Tisbe battagliai correlated positively with T-P, $\mathrm{NO}_{2}{ }^{-}$, and $\mathrm{NH}_{4}{ }^{+}$. However, halophylic copepods, dinoflagellates, Heterotrichidae, Hymenostomatidae, Pleuronematina, suspended matter, and salinity are found in the positive part of the analysis.
Fig. 3 Temporal and spatial variation of chlorophyll $a$ concentration in ponds A1, A5, A16, and C31

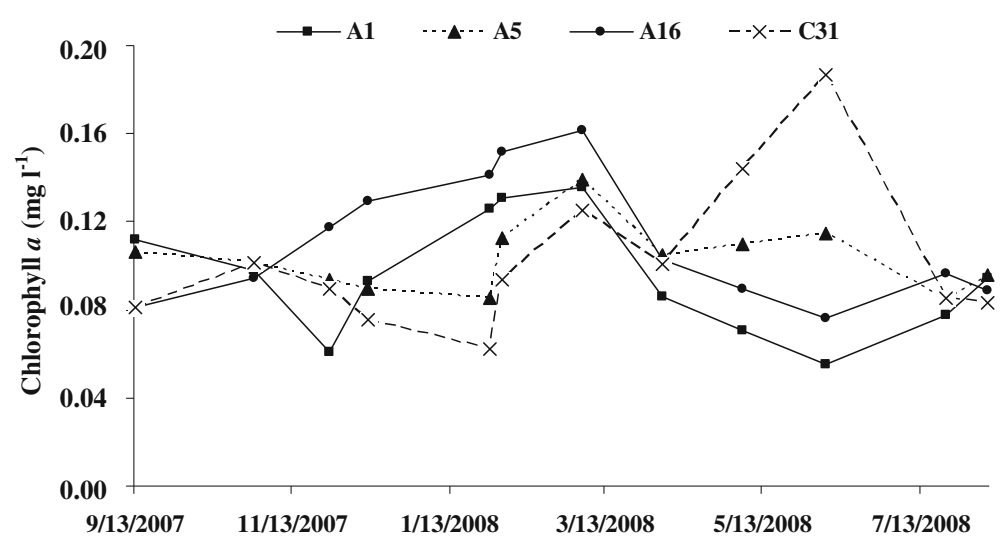


Table 3 Quantitative and structural characteristics of the ciliate community sampled in ponds A1, A5, A16, and C31

\begin{tabular}{|c|c|c|c|c|c|c|}
\hline & $\begin{array}{l}\text { Total ciliate } \\
\text { abundance } \\
\left(\times 10^{3} \text { cell } 1^{-3}\right)\end{array}$ & $\begin{array}{l}\text { Diversity index } \\
\text { (bits cell }{ }^{-1} \text { ) }\end{array}$ & Evenness & $\begin{array}{l}\text { Abundance of } \\
\text { dominant species } \\
\left(\times 10^{3} \text { cell } 1^{-3}\right)\end{array}$ & $\begin{array}{l}\text { Occurrence frequency } \\
\text { of dominant species (\%) }\end{array}$ & Dominant species \\
\hline \multicolumn{7}{|l|}{ A 1} \\
\hline $13 / 09 / 2007$ & 11 & 1.78 & 0.13 & 5 & 75 & Strombidium capitatum \\
\hline $29 / 10 / 2007$ & 2 & 1 & 0.09 & $\begin{array}{l}1 \\
1\end{array}$ & 8.33 & $\begin{array}{l}\text { Mesodinium sp. } \\
\text { S. capitatum }\end{array}$ \\
\hline $27 / 11 / 2007$ & 3 & 0.91 & 0.07 & 2 & 33.33 & Urotricha sp. \\
\hline $12 / 12 / 2007$ & 8 & 1.45 & 0.11 & 3 & 66.66 & Lohmaniella oviformis \\
\hline $28 / 01 / 2011$ & 13 & 1.98 & 0.14 & 6 & & L. oviformis \\
\hline $02 / 02 / 2011$ & 6.75 & 2.15 & 0.18 & 3 & & L. oviformis \\
\hline $04 / 03 / 2011$ & 0.5 & 2.32 & 0.25 & $\begin{array}{l}0.1 \\
0 \\
0 \\
0 \\
0\end{array}$ & $\begin{array}{l}50 \\
25 \\
25 \\
25 \\
8.33\end{array}$ & $\begin{array}{l}\text { Strombidium conicum } \\
\text { Halteria } \text { sp. } \\
\text { Leegardiella sol } \\
\text { Colpoda } \text { sp. } \\
\text { Pleuronema } \mathrm{sp} .\end{array}$ \\
\hline $04 / 04 / 2011$ & 61 & 2 & 0.12 & 24 & 16.66 & Strombidium acutum \\
\hline 05/05/2011 & 69.5 & 2.15 & 0.13 & 16 & & L. oviformis \\
\hline 06/06/2011 & 78 & 2.31 & 0.14 & 24 & 16.66 & Tintinnopsis beroidea \\
\hline $23 / 07 / 2011$ & 11 & 1.67 & 0.12 & 5 & & Leegardiella sol \\
\hline 08/08/2011 & 11 & 1.73 & 0.12 & 3 & & S. capitatum \\
\hline \multicolumn{7}{|l|}{ A5 } \\
\hline $13 / 09 / 2007$ & 6 & 1.25 & 0.09 & 4 & 75 & S. capitatum \\
\hline $29 / 10 / 2007$ & 27 & 0.5 & 0.04 & 24 & 41.66 & Urotricha sp. \\
\hline $27 / 11 / 2007$ & 3 & 1.58 & 0.09 & $\begin{array}{l}1 \\
1 \\
1\end{array}$ & 33.33 & $\begin{array}{l}\text { S. capitatum } \\
\text { Strobilidium neptuni } \\
\text { Urotricha } \mathrm{sp} .\end{array}$ \\
\hline $12 / 12 / 2007$ & 94.5 & 1.59 & 0.09 & 66 & 25 & S. conicum \\
\hline $28 / 01 / 2011$ & 186 & 1.59 & 0.09 & $\begin{array}{l}132 \\
132\end{array}$ & 83.33 & $\begin{array}{l}\text { L. oviformis } \\
\text { S. capitatum }\end{array}$ \\
\hline $02 / 02 / 2011$ & 93.1 & 1.29 & 0.09 & 66 & & S. conicum \\
\hline $04 / 03 / 2011$ & 2 & 1 & 0.09 & $\begin{array}{l}1 \\
1\end{array}$ & & $\begin{array}{l}\text { L. oviformis } \\
\text { S. capitatum }\end{array}$ \\
\hline 04/04/2011 & 1 & 0 & 0 & 1 & & L. oviformis \\
\hline $05 / 05 / 2011$ & 3.5 & 0.62 & 0.05 & 2 & & S. capitatum \\
\hline 06/06/2011 & 6 & 1.25 & 0.09 & 4 & & S. capitatum \\
\hline $23 / 07 / 2011$ & 6 & 1.79 & 0.142 & 3 & 16.66 & Tintinnidium balechi \\
\hline 08/08/2011 & 7 & 1.52 & 0.12 & 2 & & S. capitatum \\
\hline \multicolumn{7}{|l|}{ A16 } \\
\hline $13 / 09 / 2007$ & 5.7 & 0.78 & 0.06 & 4.9 & 75 & L. oviformis \\
\hline $29 / 10 / 2007$ & 12.5 & 1.85 & 0.13 & 6.5 & 66.66 & Urotricha sp. \\
\hline \multirow[t]{4}{*}{$27 / 11 / 2007$} & 4 & 2 & 0.16 & 1 & 75 & L. oviformis \\
\hline & & & & 1 & 25 & S. capitatum \\
\hline & & & & 1 & 41.66 & T. beroidea \\
\hline & & & & 1 & 66 & Urotricha sp. \\
\hline $12 / 12 / 2007$ & 11 & 1 & 0.07 & 9.5 & & Urotricha sp. \\
\hline
\end{tabular}


Table 3 (continued)

\begin{tabular}{|c|c|c|c|c|c|c|}
\hline & $\begin{array}{l}\text { Total ciliate } \\
\text { abundance } \\
\left(\times 10^{3} \text { cell } 1^{-3}\right)\end{array}$ & $\begin{array}{l}\text { Diversity index } \\
\left(\text { bits cell }{ }^{-1} \text { ) }\right.\end{array}$ & Evenness & $\begin{array}{l}\text { Abundance of } \\
\text { dominant species } \\
\left(\times 10^{3} \text { cell } 1^{-3}\right)\end{array}$ & $\begin{array}{l}\text { Occurrence frequency } \\
\text { of dominant species (\%) }\end{array}$ & Dominant species \\
\hline $28 / 01 / 2011$ & 18 & 0 & 0 & 18 & & Urotricha sp. \\
\hline $02 / 02 / 2011$ & 53.5 & 1.01 & 0.06 & 27.62 & & Urotricha sp. \\
\hline 04/03/2011 & 89 & 2.02 & 0.12 & 37.25 & & Urotricha $\mathrm{sp}$. \\
\hline 04/04/2011 & 15 & 2.28 & 0.16 & 4 & & T. beroidea \\
\hline \multirow[t]{2}{*}{ 05/05/2011 } & 13.5 & 2.23 & 0.16 & 3 & 41.66 & S. conicum \\
\hline & & & & 3 & & L. oviformis \\
\hline 06/06/2011 & 12 & 2.18 & 0.16 & 4 & & S. conicum \\
\hline \multirow[t]{3}{*}{$23 / 07 / 2011$} & 15 & 1.58 & 0.11 & 5 & & S. conicum \\
\hline & & & & 5 & 33.33 & $\begin{array}{l}\text { Strombidium } \\
\text { chlorophilum }\end{array}$ \\
\hline & & & & 5 & & Urotricha sp. \\
\hline \multirow[t]{3}{*}{ 08/08/2011 } & 10.35 & 1.18 & 0.08 & 2.5 & & S. conicum \\
\hline & & & & & & S. chlorophilum \\
\hline & & & & & & Urotricha sp. \\
\hline \multicolumn{7}{|l|}{$\mathrm{C} 31$} \\
\hline $13 / 09 / 2007$ & 39.33 & 1.48 & 0.11 & 4 & 91.66 & Fabrea salina \\
\hline $29 / 10 / 2007$ & 39.38 & 2.34 & 0.2 & 1.4 & & F. salina \\
\hline $27 / 11 / 2007$ & 39.41 & 1.97 & 0.12 & 14 & & F. salina \\
\hline $12 / 12 / 2007$ & 39.42 & 1.73 & 0.13 & 7 & & F. salina \\
\hline $28 / 01 / 2011$ & 39.47 & 1.5 & 0.11 & 8 & 25 & Aspidisca $\mathrm{sp}$. \\
\hline $02 / 02 / 2011$ & 39.48 & 1.4 & 0.12 & 4 & & Aspidisca $\mathrm{sp}$. \\
\hline 04/03/2011 & 39.51 & 1.3 & 0.11 & 1.7 & & F. salina \\
\hline $04 / 04 / 2011$ & 39.54 & 0.79 & 0.054 & 19 & & F. salina \\
\hline 05/05/2011 & 39.57 & 1.1 & 0.073 & 23 & & F. salina \\
\hline 06/06/2011 & 39.60 & 1.4 & 0.09 & 27 & & F. salina \\
\hline $23 / 07 / 2011$ & 39.65 & 0.59 & 0.04 & 6 & & F. salina \\
\hline 08/08/2011 & 39.66 & 1.04 & 0.08 & 5 & & F. salina \\
\hline
\end{tabular}

In summary, the PCA shows that copepod distribution is more susceptible to the influence of salinity, suspended matter, phytoplankton (diatoms and dinoflagellates) and nutrients than to that of temperature, chlorophyll $a$ and total ciliates. The sample score distribution (Fig. 6) indicated clear differences from one pond to another.

\section{Discussion}

In this study, the nutrient concentrations recorded in pond A1 were much lower than those reported before restoration in the same pond (Abid et al. 2008; Khemakhem et al. 2010) which is characteristic of eutrophic systems and can be attributed to anthropogenic interference in the Sfax coastal waters (Khemakhem et al. 2010). The improvement of the A1 trophic state (Table 5) is attributed to the restoration efforts undertaken during 2007 along the part of the city's shoreline in direct contact with the first of the local solar saltern ponds. We noticed a drastic decrease in T-P and phosphates from 185.8 and $145.9 \mu \mathrm{mol} \mathrm{l}^{-1}$, respectively (Abid et al. 2008), to 60.46 and $19.1 \mu \mathrm{mol} \mathrm{l}^{-1}$ as is the case in the present study. In contrast, the phosphorous concentrations in the other ponds were comparable to those of previous studies (Table 5), confirming that the phosphorus there depends more on the internal recycling processes (e.g., release from sediment, mineralization of organic matter) which are a common feature in most coastal lagoons (Abdennadher et al. 2012) 
Fig. 4 Temporal and spatial variation of copepod groups and total copepods in ponds $\mathrm{A} 1, \mathrm{~A} 5, \mathrm{~A} 16$, and $\mathrm{C} 31$ $\boxminus$ Cyclopoida 0 Calanoida $\square$ Harpacticoida Poecilostomatida $\square$ Total copepods

Abundance $\left(\times 10^{4}\right.$ ind $\left.\mathrm{m}^{-3}\right)$
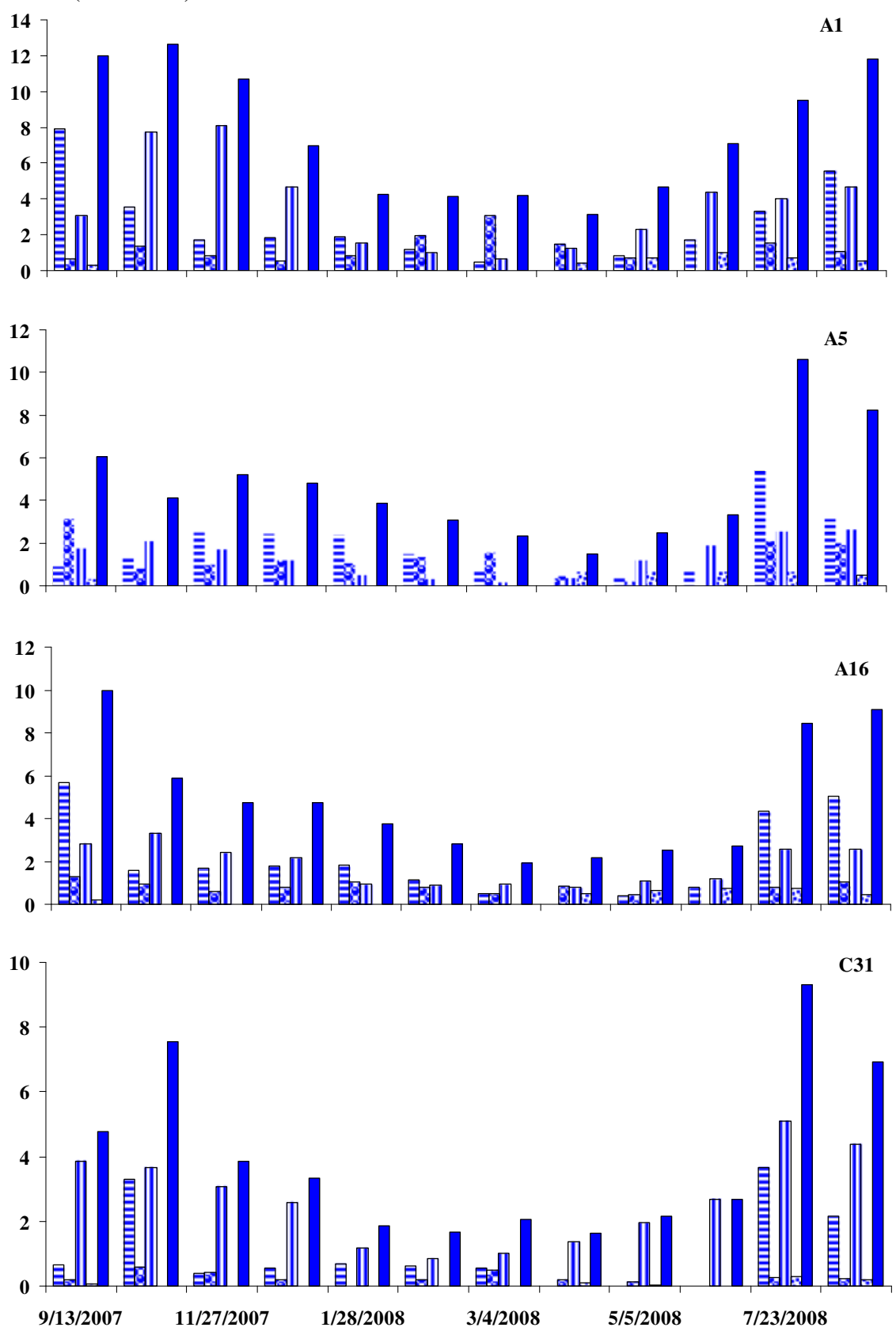

than it does on seawater inflow. In pond $\mathrm{A} 1$, we also noted a slight decrease in the relative dinoflagellate contribution from 22-43 (Khemakhem et al. 2010) to $18 \%$ as found in the present study, while that of diatoms did not change. However, within these diatoms, an increase in the diversification of the pennate species was observed after restoration (14 versus
8 species) (Abid et al. 2008) while that of centric diatoms did not change ( 8 versus 8 species). The shifts in dominance of pennate over fast-growing centric diatoms indicate a phytoplankton community under less stress than prior to restoration. Moreover, while small diatoms, especially Chaetoceros sp. and Nitzschia longissima invaded pond A1 before restoration, 
Table 4 Quantitative and structural characteristics of the copepod community sampled in ponds A1, A5, A16, and C31

\begin{tabular}{|c|c|c|c|c|c|}
\hline $\begin{array}{l}\text { Copepod total } \\
\text { abundance } \\
\left(\times 10^{4} \text { ind } \mathrm{m}^{-3}\right)\end{array}$ & $\begin{array}{l}\text { Diversity index } \\
\text { (bits ind }^{-1} \text { ) }\end{array}$ & Evenness & $\begin{array}{l}\text { Abundance of } \\
\text { dominant species } \\
\left(\times 10^{4} \text { ind } \mathrm{m}^{-3}\right)\end{array}$ & $\begin{array}{l}\text { Occurrence frequency } \\
\text { of dominant species (\%) }\end{array}$ & Dominant species \\
\hline
\end{tabular}

\begin{tabular}{|c|c|c|c|c|c|c|}
\hline \multicolumn{7}{|l|}{ A1 } \\
\hline $13 / 09 / 2007$ & 19.17 & 2.23 & 0.13 & 6.00 & 66.66 & Oithona similis \\
\hline $29 / 10 / 2007$ & 27.72 & 2.98 & 0.18 & 4.05 & 91.66 & Clytemnestra scutellata \\
\hline $27 / 11 / 2007$ & 52.00 & 2.84 & 0.17 & 2.96 & & C. scutellata \\
\hline $12 / 12 / 2007$ & 116.11 & 2.82 & 0.18 & 1.89 & & C. scutellata \\
\hline $28 / 01 / 2011$ & 180.55 & 2.50 & 0.16 & 1.33 & 91.66 & Oithona nana \\
\hline $02 / 02 / 2011$ & 94.50 & 4.38 & 0.29 & 1.46 & 50 & Paracartia grani \\
\hline 04/03/2011 & 8.59 & 2.20 & 0.14 & 2.10 & & P. grani \\
\hline $04 / 04 / 2011$ & 5.97 & 1.82 & 0.12 & 1.47 & 66.66 & Acartia clause \\
\hline $05 / 05 / 2011$ & 8.59 & 2.73 & 0.18 & 0.95 & & C. scutellata \\
\hline 06/06/2011 & 12.13 & 2.57 & 0.16 & 1.73 & 83.33 & Canuella perplexa \\
\hline $23 / 07 / 2011$ & 14.29 & 3.30 & 0.20 & 1.80 & 66.66 & O. similis \\
\hline 08/08/2011 & 17.82 & 2.99 & 0.18 & 3.90 & & O. similis \\
\hline \multicolumn{7}{|l|}{ A5 } \\
\hline $13 / 09 / 2007$ & 10.50 & 2.77 & 0.17 & 1.44 & 91.66 & Bryocamptus sp. \\
\hline $29 / 10 / 2007$ & 41.89 & 2.82 & 0.18 & 1.31 & & Bryocamptus sp. \\
\hline $27 / 11 / 2007$ & 21.35 & 2.70 & 0.17 & 1.52 & 66.66 & O. similis \\
\hline $12 / 12 / 2007$ & 14.44 & 2.59 & 0.17 & 1.25 & & O. similis \\
\hline $28 / 01 / 2011$ & 7.32 & 1.99 & 0.13 & 1.38 & 91.66 & Oithona nana \\
\hline $02 / 02 / 2011$ & 5.36 & 2.22 & 0.15 & 1.03 & 91.66 & P. grani \\
\hline 04/03/2011 & 3.50 & 1.88 & 0.13 & 1.08 & & P. grani \\
\hline $04 / 04 / 2011$ & 6.63 & 1.78 & 0.13 & 0.66 & 50 & Oncaea conifera \\
\hline 05/05/2011 & 3.74 & 2.76 & 0.19 & 0.65 & 66.66 & C. perplexa \\
\hline 06/06/2011 & 6.00 & 2.44 & 0.16 & 0.93 & & C. perplexa \\
\hline $23 / 07 / 2011$ & 87.48 & 2.70 & 0.16 & 3.75 & & O. nana \\
\hline 08/08/2011 & 49.49 & 2.99 & 0.18 & 2.13 & & O. nana \\
\hline \multicolumn{7}{|l|}{ A16 } \\
\hline $13 / 09 / 2007$ & 43.50 & 1.87 & 0.11 & 5.20 & 91.66 & O. nana \\
\hline $29 / 10 / 2007$ & 15.70 & 1.96 & 0.12 & 2.37 & 100 & Bryocamptus sp. \\
\hline $27 / 11 / 2007$ & 7.96 & 1.62 & 0.10 & 2.04 & & Bryocamptus sp. \\
\hline $12 / 12 / 2007$ & 5.21 & 2.59 & 0.17 & 1.82 & & Bryocamptus sp. \\
\hline $28 / 01 / 2011$ & 1.18 & 2.15 & 0.17 & 1.04 & 66.66 & O. similis \\
\hline $02 / 02 / 2011$ & 3.59 & 2.90 & 0.20 & 0.64 & 91.66 & O. nana \\
\hline 04/03/2011 & 4.62 & 2.38 & 0.17 & 0.68 & 83.33 & C. perplexa \\
\hline 04/04/2011 & 2.33 & 2.29 & 0.16 & 0.69 & 91.66 & P. grani \\
\hline 05/05/2011 & 2.90 & 3.19 & 0.22 & 0.46 & 50 & O. conifer \\
\hline $06 / 06 / 2011$ & 3.32 & 2.47 & 0.17 & 0.80 & & O. nana \\
\hline $23 / 07 / 2011$ & 20.30 & 3.16 & 0.19 & 2.60 & & O. nana \\
\hline 08/08/2011 & 31.81 & 2.55 & 0.15 & 3.90 & & O. nana \\
\hline \multicolumn{7}{|l|}{ C31 } \\
\hline $13 / 09 / 2007$ & 4.88 & 1.24 & 0.08 & 3.65 & 100 & Bryocamptus sp. \\
\hline $29 / 10 / 2007$ & 11.39 & 2.05 & 0.13 & 3.34 & & Bryocamptus sp. \\
\hline $27 / 11 / 2007$ & 6.24 & 1.31 & 0.09 & 2.88 & & Bryocamptus sp. \\
\hline
\end{tabular}


Table 4 (continued)

\begin{tabular}{|c|c|c|c|c|c|c|}
\hline & $\begin{array}{l}\text { Copepod total } \\
\text { abundance } \\
\left(\times 10^{4} \text { ind } \mathrm{m}^{-3}\right)\end{array}$ & $\begin{array}{l}\text { Diversity index } \\
\text { (bits ind }^{-1} \text { ) }\end{array}$ & Evenness & $\begin{array}{l}\text { Abundance of } \\
\text { dominant species } \\
\left(\times 10^{4} \text { ind } \mathrm{m}^{-3}\right)\end{array}$ & $\begin{array}{l}\text { Occurrence frequency } \\
\text { of dominant species (\%) }\end{array}$ & Dominant species \\
\hline $12 / 12 / 2007$ & 4.89 & 1.21 & 0.08 & 2.49 & & Bryocamptus sp. \\
\hline 28/01/2011 & 2.60 & 0.95 & 0.07 & 1.18 & & Bryocamptus sp. \\
\hline 02/02/2011 & 2.72 & 1.98 & 0.14 & 0.63 & & Bryocamptus sp. \\
\hline 04/03/2011 & 3.31 & 2.47 & 0.17 & 0.56 & & Bryocamptus sp. \\
\hline 04/04/2011 & 4.52 & 1.67 & 0.12 & 1.10 & 58.33 & Mesochra lilljeborgi \\
\hline 05/05/2011 & 5.00 & 1.78 & 0.12 & 0.98 & & M. lilljeborgi \\
\hline 06/06/2011 & 5.60 & 1.25 & 0.08 & 1.60 & & Bryocamptus sp. \\
\hline 23/07/2011 & 16.20 & 2.48 & 0.15 & 2.50 & & Bryocamptus sp. \\
\hline 08/08/2011 & 10.12 & 2.33 & 0.14 & 2.96 & & Bryocamptus sp. \\
\hline
\end{tabular}

they now appeared to share the total diatom biomass with larger diatom species namely Thalassiosira sp. and Navicula sp. Since the hydrographic conditions such as water turbulence remained nearly the same in pond A1 over the years, the appearance of large diatoms may be attributed to decreased phosphorus inputs resulting from restoration. Indeed, it is known that as phosphorus concentration decreases large diatoms develop a greater competitive advantage for $\mathrm{P}$ assimilation (Reynolds 1997). This confirms the findings of previous investigations in coastal waters showing that nutrients are the main resource, with temperature as the main seasonal signal for phytoplankton growth since the salinity level is still near that of seawater. The restoration of the coastal waters therefore broadens the spectrum of existing conditions, enabling the coexistence of a great number of species. The present findings provided information on the direct impact of restoration on the phytoplankton community and demonstrated the suitability of using phytoplankton as bioindicators of environmental stress (Aleya et al. 2011). This is also confirmed by the diversification observed within the ciliate community, namely Oligotrichida, with 19 new taxa recorded during this study though they were missing in the previous study by Elloumi et al. (2009). Among these new Oligotrichida taxa, we found Strombidium capitatumis to be a bioindicator of less stressed marine ecosystems (Jiang et al. 2011). High species ciliate diversity is commonly observed in marine systems of low organic pollution (Ismael and Dorgham 2003) and in less stressed marine sites (Jiang et al. 2011). The copepod composition in the Sfax saltern is comparable to other Mediterranean coastal environments, with, however, an evident prevalence of halophylic species in adaptation to the rising salt concentration (Abdennadher et al. 2012; Badosa et al. 2006; Hannachi et al. 2011). During this study, we found ten species in pond A1 cited for the first time in the Sfax solar saltern, compared with the findings of Toumi et al. (2005) in their previous study period of 1999-2000. Among them were large species such as Centropages kroyeri and Clytemnestra scutellata, known to indicate a good ecosystem status (Kambursha and Fonda-Umani 2006) and/or a modification in the prey spectrum (Calbet et al. 2001). We infer, with regard to food resources represented by phytoplankton and available data on ciliates, that the increase in the size of potential prey as seen in our study may also be an important driver of zooplankton community structure in the saltern. Previous studies along the coast of Sfax (Drira et al. 2010; Rekik et al. 2012) and in other marine systems (Verity and Smetacek 1996; Rollwagen-Bollens et al. 2011) indicate that the quantity and quality of specific types of food would be expected to affect variation in zooplankton composition. We also previously found in laboratory experiments that copepods preferred large ciliates when these were abundant (Hartmann et al. 1993). Copepods in ponds A1 and A5 (salinity, <50) were mainly thalassophylic. A slight increase in salinity in A1 from adjacent seawater positively influenced total copepod abundance. Christou (1998) also reported the importance of salinity as the most significant factor affecting the inter-annual variability of copepod abundances in Mediterranean coastal areas with a salinity of $39 \mathrm{psu}$. In addition, the salinity of A1 may be optimal for the egg production of dominant copepods and the hatching success of their remaining eggs (Peck and Holste 
Fig. 5 Seasonal variation of species abundances and $\mathrm{H}^{\prime}$ in ponds $\mathrm{A} 1, \mathrm{~A} 5, \mathrm{~A} 16$, and C31

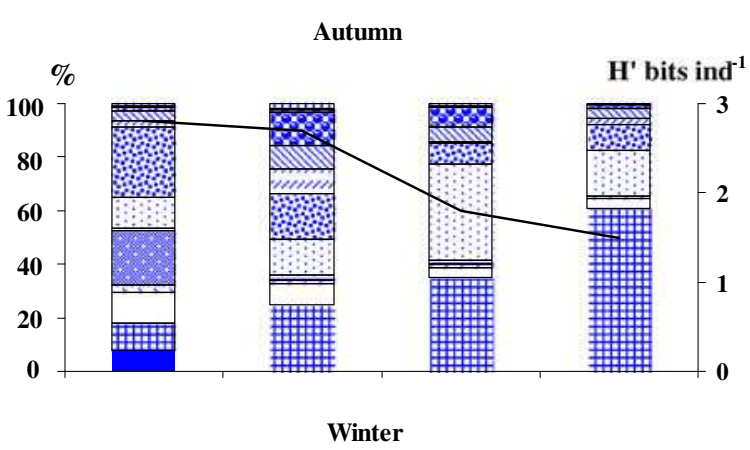

Oncaea mediterranea

핌 Oncaea conifera

든도 Centropages ponticus

$\square$ Acartia longiremis

Paracartia latisetosa

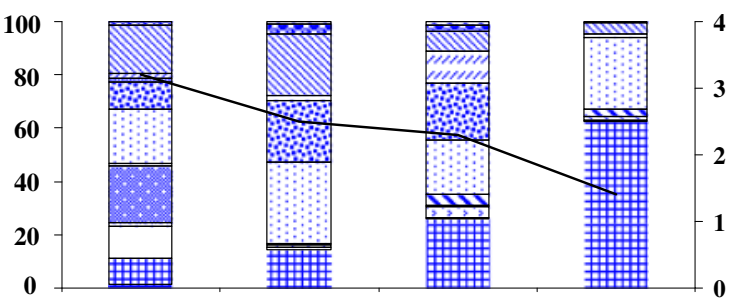

Paracartia grani

Acartia clausi

$\square$ Cyclopina sp.

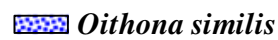

Oithona nana

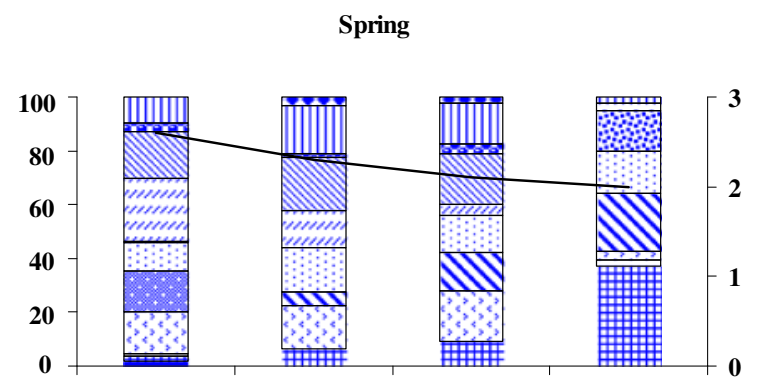

Mesochra lilljeborgi

可icrosetella norvegica

$\square$ Clytemnestra scutellata

Canuella perplexa

Tisbe battagliai

Summer

姍冊 Bryocamptus sp.

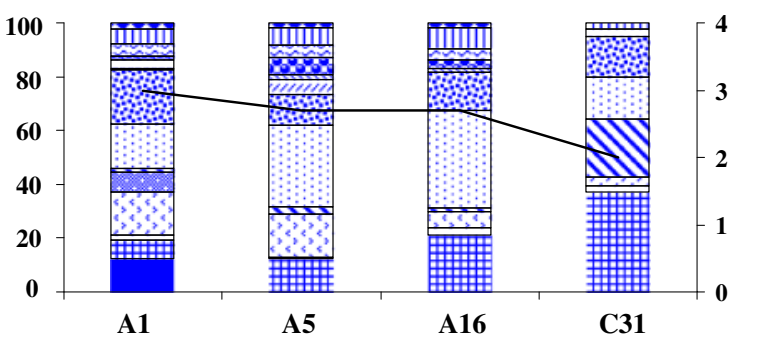

Euterpina acutifrons
2006). However, this was not the case for pond A5 because the increase in salt concentration negatively affected copepod abundance leading to complete absence of C. scutellata, Euterpina acutifrons, and Cyclopina sp. These three taxa have been shown to persist in both coastal marine waters (Annabi-Trabelsi 2006; Drira et al. 2010) and within the polyhaline zone (37> surface salinity $>18$ psu) of estuaries (Hoffmeyer and Barria de Cao 2007; Chertoprud et al. 2009). In ponds $A 1$ and A5, thalassophylic copepods are forced to face salinity stress and an important seasonal temperature variation (the difference between the hot and the cold season is greater than $11^{\circ} \mathrm{C}$ ) implying a trade-off between fighting osmotic stress and improvement of food availability. With the increase in salinity to $>50 \mathrm{psu}$ in A16 and C21, temperature positively affected total copepod abundances, thus $O$. nana dominated at A5 and A16 as it is an euryhaline and eurythermal species. The halophylic 
Fig. 6 PCA performed on biological parameters and different environmental factors in four ponds during 1 year of study
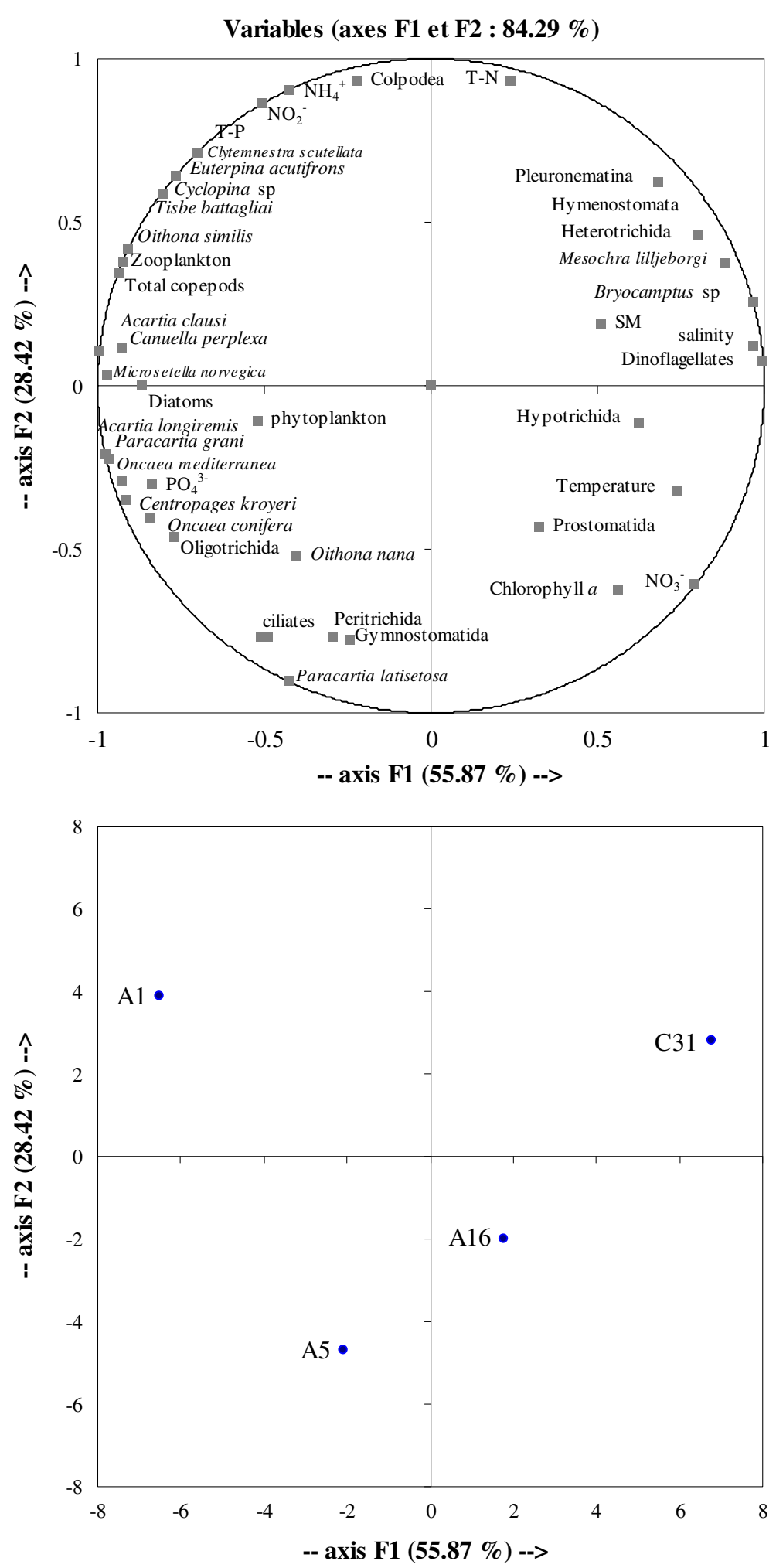

taxa Bryocamptus sp. and $M$. lilljeborgi increased gradually in A16 and peaked in pond C31, their demography showing positive correlations with salinity. 
Table 5 Min, max and mean \pm SD of physicochemical and biological variables at the first pond A1 before and after restoration

\begin{tabular}{|c|c|c|c|c|c|c|c|}
\hline \multirow[t]{2}{*}{ Physicochemical and biological parameters } & \multicolumn{3}{|c|}{ Before restoration } & \multicolumn{3}{|c|}{ Present work (2007-2008) } & \multirow[t]{2}{*}{$F$ values $(d f)$} \\
\hline & Min & Max & Mean \pm SD & Min & Max & Mean \pm SD & \\
\hline \multicolumn{8}{|l|}{ Physical parameters } \\
\hline Temperature $\left({ }^{\circ} \mathrm{C}\right)$ & 12.6 & 32.3 & $24.5 \pm 6.3$ & 11.5 & 29 & $20.7 \pm 5.57$ & $0.91(23)$ \\
\hline Salinity (psu) & 40.2 & 60.4 & $45.0 \pm 5.4$ & 38 & 45.2 & $41.54 \pm 2.38$ & $4.64(23)^{*}$ \\
\hline $\mathrm{pH}$ & 6.11 & 8.18 & $7.2 \pm 0.7$ & 7.01 & 8.93 & $7.71 \pm 0.52$ & $2.13(23)$ \\
\hline Suspended matter $\left(\mu \mathrm{g} \mathrm{l}^{-1}\right)$ & 32 & 285.75 & $130.9 \pm 96.6$ & 105 & 825 & $399.37 \pm 217.83$ & $42.64(23)^{* * *}$ \\
\hline \multicolumn{8}{|l|}{ Chemical parameters } \\
\hline $\mathrm{NO}_{3}^{-}\left(\mu \mathrm{mol} \mathrm{l}{ }^{-1}\right)$ & 1.79 & 74.24 & $45.6 \pm 32.8$ & 1.79 & 15.41 & $6.52 \pm 3.73$ & $3.02(23)$ \\
\hline $\mathrm{NO}_{2}^{-}\left(\mu \mathrm{mol} \mathrm{l} 1^{-1}\right)$ & 0.02 & 38.98 & $9.29 \pm 12.42$ & 0.02 & 0.72 & $0.25 \pm 0.21$ & $6.36(23)^{*}$ \\
\hline $\mathrm{NH}_{4}^{+}\left(\mu \mathrm{mol} 1^{-1}\right)$ & 0.47 & 43.33 & $32.8 \pm 6.3$ & 0.47 & 15.01 & $5.21 \pm 5.81$ & $5.29(23)^{*}$ \\
\hline Total nitrogen $\left(\mu \mathrm{mol} 1^{-1}\right)$ & 12.09 & 771.43 & $322.0 \pm 205.8$ & 2.78 & 26.75 & $16.36 \pm 7.73$ & $13.21(23)^{* *}$ \\
\hline $\mathrm{PO}_{4}{ }^{3-}\left(\mu \mathrm{mol} \mathrm{l} 1^{-1}\right)$ & 1.06 & 285.11 & $145.9 \pm 110.0$ & 2.82 & 39.96 & $19.19 \pm 11.95$ & $5.59(23)^{*}$ \\
\hline Total phosphate $\left(\mu \mathrm{mol} 1^{-1}\right)$ & 5.33 & 363.83 & $185.8 \pm 132.8$ & 13.25 & 201.48 & $60.46 \pm 53.46$ & $7.67(23)^{* *}$ \\
\hline $\mathrm{Si}(\mathrm{OH})_{4}\left(\mu \mathrm{mol} 1^{-1}\right)$ & 3.63 & 43.63 & $12.71 \pm 11.29$ & 2.46 & 43.64 & $12.65 \pm 13.84$ & $0.0001(23)$ \\
\hline $\mathrm{N} / \mathrm{P}$ ratio & & & 0.8 & 0.139 & 7.87 & $1.92 \pm 2.89$ & $0.71(23)$ \\
\hline \multicolumn{8}{|l|}{ Biological parameters } \\
\hline Chlorophyll $a$ concentration $\left(\mathrm{mg} \mathrm{l}^{-1}\right)$ & 0.002 & 0.19 & $0.032 \pm 0.051$ & 0.05 & 0.13 & $0.09 \pm 0.02$ & $10.04(23)^{* *}$ \\
\hline Total phytoplankton density $\left(\times 10^{3}\right.$ cells $\left.1^{-1}\right)$ & 34.4 & 2,096 & $600 \pm 800$ & 29 & 142.7 & $78.31 \pm 34.96$ & $8.71(23)^{* *}$ \\
\hline Cyanobacteriae density $\left(\times 10^{3}\right.$ cells $\left.1^{-1}\right)$ & & & & 0 & 21 & $1.85 \pm 6.03$ & \\
\hline Diatoms density $\left(\times 10^{3}\right.$ cells $\left.1^{-1}\right)$ & 0 & 1,988 & $302.80 \pm 603.09$ & 22.8 & 102.4 & $50.2 \pm 22.54$ & $2.10(23)$ \\
\hline Dinophyceae density $\left(\times 10^{3}\right.$ cells $\left.1^{-1}\right)$ & 0 & 2,072 & $444.48 \pm 702.03$ & 0 & 40.3 & $14.05 \pm 12.77$ & $4.5(23)^{*}$ \\
\hline Euglenophyceae density $\left(\times 10^{3}\right.$ cells $\left.1^{-1}\right)$ & 0 & 96 & $13.59 \pm 29.49$ & 0 & 35 & $12.20 \pm 15.08$ & $0.02(23)$ \\
\hline Silicoflagellate density $\left(\times 10^{3}\right.$ cells $\left.1^{-1}\right)$ & - & - & - & 0 & 14 & $1.75 \pm 4.35$ & \\
\hline Total ciliate density $\left(\times 10^{3}\right.$ cells $\left.1^{-1}\right)$ & 0.9 & 63.8 & $16.075 \pm 19.3$ & 0.5 & 78 & $22.89 \pm 28.6$ & $0.46(23)$ \\
\hline Oligotrichidae density $\left(\times 10^{3}\right.$ cells $\left.1^{-1}\right)$ & 0.6 & 63.4 & $11.41 \pm 18.44$ & 0.3 & 76 & $21.66 \pm 28.67$ & $1.08(23)$ \\
\hline Colpodea density $\left(\times 10^{3}\right.$ cells $\left.1^{-1}\right)$ & - & - & - & 0 & 1 & $0.13 \pm 0.3$ & \\
\hline Gymnostomatidae density $\left(\times 10^{3}\right.$ cells $\left.1^{-1}\right)$ & 0 & 0.8 & $0.15 \pm 0.31$ & 0 & 1 & $0.08 \pm 0.28$ & $0.18(23)$ \\
\hline Prostomatidae density $\left(\times 10^{3}\right.$ cells $\left.1^{-1}\right)$ & 0 & 31.6 & $3.99 \pm 9.51$ & 0 & 4 & $0.75 \pm 1.28$ & $3.19(23)$ \\
\hline Pleuronematina density $\left(\times 10^{3}\right.$ cells $\left.1^{-1}\right)$ & - & - & - & 0 & 0.1 & $0.012 \pm 0.03$ & \\
\hline Heterotrichida density $\left(\times 10^{3}\right.$ cells $\left.1^{-1}\right)$ & 0 & 0.1 & $0.008 \pm 0.02$ & - & - & - & \\
\hline Hymenostomatida density $\left(\times 10^{3}\right.$ cells $\left.1^{-1}\right)$ & 0 & 0.4 & $0.09 \pm 0.16$ & - & - & - & \\
\hline Hypotrichida density $\left(\times 10^{3}\right.$ cells $\left.1^{-1}\right)$ & 0 & 0.4 & $0.13 \pm 0.18$ & 0 & 2 & $0.25 \pm 0.62$ & $0.18(23)$ \\
\hline Total zooplankton density $\left(\times 10^{3}\right.$ ind $\left.\mathrm{m}^{-3}\right)$ & & & $160 \pm 270$ & 52.46 & 2001.75 & $707.62 \pm 754.98$ & $10.10(23)^{* *}$ \\
\hline Total copepod density $\left(\times 10^{3}\right.$ ind $\left.\mathrm{m}^{-3}\right)$ & & & $106 \pm 193$ & 59.65 & 1805.54 & $464.52 \pm 554.39$ & \\
\hline Cyclopoid density $\left(\times 10^{3}\right.$ ind $\left.\mathrm{m}^{-3}\right)$ & & & $9 \pm 1.8$ & 0 & 79.2 & $24.94 \pm 22.86$ & \\
\hline Calanoid density $\left(\times 10^{3}\right.$ ind $\left.\mathrm{m}^{-3}\right)$ & & & $8.2 \pm 3.1$ & 0 & 30.82 & $11.64 \pm 7.99$ & \\
\hline Harpacticoid density $\left(\times 10^{3}\right.$ ind $\left.\mathrm{m}^{-3}\right)$ & & & $1.6 \pm 3,8$ & 6.52 & 81.19 & $36.13 \pm 24.77$ & \\
\hline Poecilostomatoid density $\left(\times 10^{3}\right.$ ind $\left.\mathrm{m}^{-3}\right)$ & & & - & 0 & 10 & $3.05 \pm 3.58$ & \\
\hline Other zooplankton density $\left(\times 10^{3}\right.$ ind $\left.\mathrm{m}^{-3}\right)$ & & & $51 \pm 48$ & 15.29 & 1252.48 & $243.09 \pm 369.02$ & \\
\hline
\end{tabular}

In the last column, results of one-way ANOVA analysis

${ }^{*} p<0.05 ;{ }^{* *} p<0.01 ; * * * p<0.001$, significant difference between sampled stations 


\section{Conclusions}

This study presents an original survey of a planktonic community at a site that offers a unique environment with high annual reproducibility of seasonal dynamics in water ponds. The choice of the fine range of salinity (41-77 psu), added to the semi-arid climate and the strict operating conditions imposed by the salt producing company, combine to render the physicochemical variables of each pond highly stable from one year to the next. This, in turn, enhances our ability to uniquely identify the influence of phosphogypsum residue reduction on the seasonal distribution, composition and dynamics of planktonic communities, chiefly at four ponds featuring a salinity gradient. The restoration of the phosphogypsum landfill not only positively influenced the biological communities under study but also the quality of the salt produced as reported by the Franco-Tunisian salt company COTUSAL.

Acknowledgments The authors would like to thank the COTUSAL staff for granting them access to the saltern and permission to take samples. This study is a part of the Ph.D. of Rayda Kobbi and was supported by The Plankton and Microbiology of Aquatic Ecosystems Laboratory (University of Sfax, Tunisia) and Chrono-Environment, UMR CNRS 6249 (University of Franche-Comté, France).

\section{References}

Abdennadher, M., Hamza, A., Fekih, W., Hannachi, I., ZouariBelaaj, A., Bradai, N. \& Aleya, L. (2012). Factors determining the dynamics of toxic blooms of 1 Alexandrium minutum during a 10- year study along the shallow southwestern Mediterranean coasts. Estuarine and Coastal Shelf Science. doi:10.1016/j.ecss.2012.04.029.

Abid, O., Sellami-Kammoun, A., Ayadi, H., Drira, Z., Bouain, A., \& Aleya, L. (2008). Biochemical adaptation of phytoplankton to salinity and nutrient gradients in a coastal solar saltern, Tunisia. Estuarine and Coastal Shelf Science, 80, 391-400.

Aleya, L., Dauta, A., \& Reynolds, C. S. (2011). Endogenous regulation of the growth-rate responses of a springdwelling strain of the freshwater alga, Chlorella minutissima, to light and temperature. European Journal of Protistology, 47, 239-244.

Annabi-Trabelsi, N., Daly-Yahia, M. N., Romdhane, M. S., \& Ben Maïz, N. (2006). Seasonal variability of planktonic copepods in Tunis North Lagoon (Tunisia, North Africa). Cahiers de Biologie Marine, 46, 325-333.

APHA. (2012). Standard methods for examination of water and waste water, 22nd. Washington: American Public Health Association.

Badosa, A., Boix, D., Brucet, S., Lopez-Flores, R., \& Quintana, X. D. (2006). Nutrients and zooplankton composition and dynamics in relation to the hydrological pattern in a confined Mediterranean salt marsh (NE Iberian Peninsula). Estuarine, Coastal and Shelf Science, 66, 513-622.

Calbet, A., Garrido, S., Saiz, E., Alcaraz, M., \& Duarte, C. M. (2001). Annual zooplankton succession in coastal NW Mediterranean waters: the importance of the smaller size fractions. Journal of Plankton Research, 23, 319-331.

Chertoprud, E. S., Gómez, S., \& Gheerardyn, H. (2009). Harpacticoida (Copepoda) fauna and the taxocene diversity of the South China Sea. Oceanology, 49, 488-498.

Christou, E. D. (1998). Interannual variability of copepods in a Mediterranean coastal area: Saronikos Gulf, Aegean Sea. Journal of Marine Systems, 15, 523-532.

Davis, J. S. (1990). Biological management for the production of salt from seawater. In I. Akatsuka (Ed.), Introduction to applied phycology (pp. 479-488). The Hague: SPB Academic Publishing BV.

Davis, J. S. (2000). Structural, function, and management of the biological system for seasonal solar saltworks. Global NEST: The International Journal, 2, 217-226.

Drira, Z., Belhassen, M., Ayadi, H., Hamza, A., Zarrad, R., Bouaïn, A., \& Aleya, L. (2010). Copepod community structure related to environmental factors from a summer cruise in the Gulf of Gabes (Tunisia, Eastern Mediterranean Sea). Journal of the Marine Biological Association of the United Kingdom, 90, 145-157.

Elevi-Bardavid, R., Mana, L., \& Oren, A. (2007). Haloplanus natans gen. nov., sp. nov., an extremely halophilic gasvacuolate archaeon from Dead Sea-Red Sea water mixtures in experimental mesocosms. International Journal of Systematic and Evolutionary Microbiology, 57, 780-783.

Elloumi, J., Guermazi, W., Ayadi, H., Bouaïn, A., \& Aleya, L. (2008). Detection of water and sediments pollution of an arid Saltern (Sfax, Tunisia) by coupling the distribution of microorganisms with hydrocarbons. Water, Air, and Soil Pollution, 187, 157-171.

Elloumi, J., Guermazi, W., Ayadi, H., Bouaïn, A., \& Aleya, L. (2009). Abundance and biomass of prokaryotic and eukaryotic microorganisms coupled with environmental factors in an arid multi-pond solar saltern (Sfax, Tunisia). Journal of the Marine Biological Association of the United Kingdom, 89, 243-253.

Hannachi, I., Drira, Z., Bel HAssen, M., Hamza, H., Ayadi, H., \& Aleya, L. (2011). Distribution of microplankton in relation to environmental variables during summer stratification in the Gulf of Hammamet, Tunisia. Journal of the Marine Biological Association of the United Kingdom, 91, 1429-1442.

Hartmann, H. J., Taleb, H., Aleya, L., \& Lair, N. (1993). Predation on ciliates by the suspension-feeding calanoid copepod Acanthodiaptomus denticornis. Canadian Journal of Fisheries and Aquatic Sciences, 50, 1382-1393.

Hoffmeyer, M. S., \& Barría de Cao, M. S. (2007). Zooplankton assemblages from a tidal channel in the Bahia Blanca estuary, Argentina. Brazilian Journal of Oceanography, 55, 97-107.

Ismael, A. A., \& Dorgham, M. M. (2003). Ecological indices as a tool for assessing pollution in El-Dekhaila Harbour (Alexandria, Egypt). Oceanologia, 45, 121-131.

Javor, B. J. (1989). Hypersaline environments. Microbiology and biogeochemistry. New York: Springer.

Jiang, Y., Xu, H., Hu, X., Zhu, M., Al-Rasheid, K. A. S., \& Warren, A. (2011). An approach to analyzing spatial 
patterns of planktonic ciliate communities for monitoring water quality in Jiaozhou Bay, northern China. Marine Pollution Bulletin, 62, 227-235.

Joint, I., Henrikson, P., Garde, K., \& Riemann, B. (2002). Primary production, nutrient assimilation and microzooplankton grazing along a hypersaline gradient. Microbial Ecology, 39, 245-257.

Kambursha, L., \& Fonda-Umani, S. (2006). Long-term copepod dynamics in the Gulf of Trieste (Northern Adriatic Sea): recent changes and trends. Climate Research, 31, 195-203.

Khemakhem, H., Elloumi, J., Moussa, M., Aleya, L., \& Ayadi, H. (2010). The concept of ecological succession applied to phytoplankton over four consecutive years in five ponds featuring a salinity gradient. Estuarine, Coastal and Shelf Science, 88, 33-44.

Oren, A. (2005). A hundred years of Dunaliella research: 19052005. Saline Systems, 10, 1186-1746.

Peck, M. A., \& Holste, L. (2006). Effects of salinity, photoperiod and adult stocking density on egg production and egg hatching success in Acartia tonsa (Calanoida: Copepoda): optimizing intensive cultures. Aquaculture, 255, 341-350.

Rekik, A., Drira, Z., Guermazi, W., Elloumi, J., Maalej, S., Aleya, L., \& Ayadi, H. (2012). Impacts of an uncontrolled phosphogypsum dumpsite on summer distribution of phytoplankton, copepods and ciliates in relation to abiotic variables along the near-shore of the southwestern Mediterranean coast. Marine Pollution Bulletin, 64, 336-346.

Reynolds, C. S. (1997). Vegetation processes in the pelagic: a model for ecosystem theory. Oldendorf/Luhe: Ecology Institute.

Rollwagen-Bollens, G. C., Gifford, S. M., \& Bollens, S. M. (2011). The role of protistan microzooplankton in the upper San Francisco estuary planktonic food web: source or sink? Estuaries and Coasts, 10, 1007-12237.

Shannon, C. E., \& Weaver, G. (1949). The Mathematical Theory of Communication. University of Illinois Press: Urbana, Chicago, IL.

Strickland, J. D. H., \& Parsons, T. R. (1968). A practical handbook of seawater analysis. Pigment analysis. Bulletin 167. Ottawa: Fisheries Research Board of Canada.

Toumi, N., Ayadi, H., Abid, O., Carrias, J. F., Sime-Ngando, T., Boukhris, M., \& Bouaîn, A. (2005). Zooplankton distribution in four ponds of different salinity: a seasonal study in the solar salterns of Sfax (Tunisia). Hydrobiologia, 534, 1-9.

Uthermöhl, H. (1958). Zur Vervollkommung der quantitativen Phytoplankton Methodik. Mitteilungen Internationale Vereinigung für Theoretische und Angewandte Limnologie, 9, 1-38.

Verity, P. G., \& Smetacek, V. (1996). Organism life cycles, predation, and the structure of marine pelagicecosystems. Marine Ecology Progress Series, 130, 277-293. 